PROCEEDINGS OF THE

AMERICAN MATHEMATICAL SOCIETY

Volume 134, Number 10, October 2006, Pages 2923-2930

S 0002-9939(06)08500-5

Article electronically published on April 11, 2006

\title{
CHARACTERIZATIONS OF ISOMETRIC ISOMORPHISMS BETWEEN UNIFORM ALGEBRAS VIA NONLINEAR RANGE-PRESERVING PROPERTIES
}

\author{
OSAMU HATORI, TAKESHI MIURA, AND HIROYUKI TAKAGI \\ (Communicated by Jonathan M. Borwein) \\ Dedicated to Professor Sin-Ei Takahasi on his sixtieth birthday
}

\begin{abstract}
Let $T$ be a surjective mapping from a uniform algebra $A$ on a compact Hausdorff space $X$ onto a uniform algebra on a compact Hausdorff space $Y$. Suppose that $f g(X)=T(f) T(g)(Y)$ holds for every $f, g \in A$. Then we have that $T$ is an almost isometric isomorphism, which is a generalization of results of Molnár (2002) and Rao and Roy (2005).
\end{abstract}

\section{INTRODUCTION}

In this paper we consider spectrum-preserving mappings on Banach algebras, mainly of complex-valued continuous functions on compact Hausdorff spaces. Although such mappings are diversely possible except linearity, spectrum-preserving linear operators between Banach algebras are studied in connection with Kaplansky's problem. On the other hand, under additional assumptions spectrum-preserving mappings are linear and multiplicative. For example, by a simple application of a theorem of Kowalski and Słodkowski 2, a mapping $T$ from a Banach algebra $A$ into a semi-simple commutative Banach algebra $B$ is linear and multiplicative if the spectrum of $T(f)-T(g)$ is included in the spectrum of $f-g$ for every pair $f$ and $g$ in $A$. On the other hand Molnár deduced linearity and multiplicativity for certain spectrum-preserving mappings on the algebra $C(\mathcal{X})$ of all complex-valued continuous functions on a first countable compact Hausdorff space $\mathcal{X}$ (Theorems 5 and 6 in [3]). In these cases the mappings $T$ are assumed to satisfy weak multiplicativity conditions such as $T(f) T(g)(\mathcal{X})=f g(\mathcal{X})$ or $(\overline{T(f)} T(g))(\mathcal{X})=\bar{f} g(\mathcal{X})$. One can easily see that a weaker assumption $(T(f) T(g))(\mathcal{X}) \subset f g(\mathcal{X})$ is not sufficient for additivity of the mappings for certain $\mathcal{X}$. Recall that a uniform algebra $A$ on a compact Hausdorff space $X$ is a closed subalgebra of $C(X)$ which separates the points of $X$ and contains constant functions. We show a generalization of a theorem of Molnár [3, Theorem 5] for the case of uniform algebras on arbitrary compact Hausdorff spaces. In section 3 we also show that the first countability for $X$, which

Received by the editors April 22, 2005.

2000 Mathematics Subject Classification. Primary 46J10, 47B48.

Key words and phrases. Uniform algebras, isometric isomorphisms, spectrum preserving map.

The authors were partially supported by the Grants-in-Aid for Scientific Research, The Ministry of Education, Science, Sports and Culture, Japan. 
is assumed in Theorem 6 of [3], can be removed. In Molnár's proofs of Theorems 5 and 6 in [3], order preservingness of the mapping is a crucial thing. For general uniform algebras, the order is out of sense and our idea of the proofs of Theorems 1.1 and 3.6 are different from that of Molnár's.

We denote the maximal ideal space of $A$ by $M_{A}$. For each $f \in A, \widehat{f}$ denotes the Gelfand transform of $f$. The supremum norm is denoted by $\|\cdot\|$. Our main result is the following. See also Theorem 3.6.

Theorem 1.1. Let $A$ and $B$ be uniform algebras on compact Hausdorff spaces $X$ and $Y$, respectively. If a mapping $T$ from $A$ onto $B$ satisfies

$$
((T f)(T g))(Y)=(f g)(X) \quad(f, g \in A),
$$

then there exist a function $\psi \in B$ with range in $\{-1,1\}$ and a homeomorphism $\varphi$ from $M_{B}$ onto $M_{A}$ such that

$$
\widehat{T f}(y)=\widehat{\psi}(y) \widehat{f}(\varphi(y)) \quad\left(y \in M_{B}\right)
$$

for all $f \in A$.

The referee notified the authors that the recent paper of Rao and Roy 4 treats the same problem for a special kind of function algebra. The authors thank the referee for valuable comments.

\section{Preliminaries}

For $r>0$, we write $D_{r}=\{z \in \mathbb{C}:|z|<r\}$. Let us consider a function whose range contains 1 and is contained in $D_{1} \cup\{1\}$. Such a function is often called a peak function. In this section, we are concerned with the peak functions in a uniform algebra $A$ on a compact Hausdorff space $X$. See [1] for a good reference for the therory of uniform algebras. By $\operatorname{Ch}(A)$, we denote the Choquet boundary of $A$. For each $x \in \mathrm{Ch}(A)$, put

$$
P_{x}=\left\{u \in A: u(X) \subset D_{1} \cup\{1\}, u(x)=1\right\} .
$$

Since every point $x$ in $\operatorname{Ch}(A)$ is a peak point in the weak sense for $A$, it follows that $P_{x}$ is nonempty. If $x_{0} \in \mathrm{Ch}(A)$, if $F$ is a closed subset of $X$ with $x_{0} \notin F$ and if $\varepsilon>0$, then there exists a $u \in P_{x_{0}}$ such that $|u(x)|<\varepsilon$ for $x \in F$.

Lemma 2.1. Let $x, y \in \operatorname{Ch}(A)$. If $P_{x} \subset P_{y}$, then $x=y$.

Proof. Assume $x \neq y$. By using the fact mentioned above, we find a $u \in P_{x}$ such that $|u(y)|<1$. Then $u \in P_{x} \backslash P_{y}$ and so $P_{x} \not \subset P_{y}$, concluding the proof.

Put $\mathcal{P}=\bigcup_{x \in \mathrm{Ch}(A)} P_{x}$. In other words, $\mathcal{P}$ is the set of all peak functions in $A$. For each $f \in \mathcal{P}$, we write

$$
K_{f}=\{x \in X: f(x)=1\} .
$$

Lemma 2.2. Let $f, g \in \mathcal{P}$. Then $K_{f} \subset K_{g}$ if and only if $1 \in(g u)(X)$ for each $u \in \mathcal{P}$ with $1 \in(f u)(X)$.

Proof. Suppose $K_{f} \subset K_{g}$ and assume that $u \in \mathcal{P}$ satisfies $1 \in(f u)(X)$. Then $f(x) u(x)=1$ for some $x \in X$. Since $f(X)$ and $u(X)$ are contained in $D_{1} \cup\{1\}$, it follows that $f(x)=u(x)=1$. Hence $x \in K_{f} \subset K_{g}$. This means $g(x)=1$ and so $g(x) u(x)=1$. Thus $1 \in(g u)(X)$, and the "only if" part is proved. 
Suppose $K_{f} \not \subset K_{g}$. Then we can find an $x_{0} \in\left(K_{f} \backslash K_{g}\right) \cap \mathrm{Ch}(A)$. Since $K_{g}$ is a closed set and $x_{0} \notin K_{g}$, there is a $u \in P_{x_{0}}$ such that $|u(x)|<1$ for every $x \in K_{g}$. We have

$$
1 \in(f u)(X) \text { and } 1 \notin(g u)(X) \text {, }
$$

because $f\left(x_{0}\right) u\left(x_{0}\right)=1$ and $g(x) u(x) \in D_{1}$ for all $x \in X$. The "if" part is proved.

Lemma 2.3. Let $f \in A$ and $x_{0} \in \operatorname{Ch}(A)$. If $\lambda=f\left(x_{0}\right)$ and $\lambda \neq 0$, then there exists a $u \in P_{x_{0}}$ such that $(1 / \lambda) f u \in P_{x_{0}}$.

Proof. Put $F_{0}=\{x \in X:|f(x)-\lambda| \geq|\lambda| / 2\}$ and

$$
F_{n}=\left\{x \in X: \frac{|\lambda|}{2^{n+1}} \leq|f(x)-\lambda| \leq \frac{|\lambda|}{2^{n}}\right\} \quad(n=1,2, \ldots) .
$$

Clearly, $F_{0}, F_{1}, \ldots, F_{n}, \ldots$ are all closed subsets of $X$ which do not contain $x_{0}$. Hence there exist $u_{0}, u_{1}, \ldots, u_{n}, \ldots \in P_{x_{0}}$ such that

$$
\begin{aligned}
& \left|u_{0}(x)\right|<\frac{|\lambda|}{\|f\|} \quad \text { for } x \in F_{0}, \\
& \left|u_{n}(x)\right|<\frac{1}{2^{n}+1} \quad \text { for } x \in F_{n} \quad(n=1,2, \ldots) .
\end{aligned}
$$

Now put

$$
u=u_{0} \sum_{k=1}^{\infty} \frac{u_{k}}{2^{k}} .
$$

The above series is majorized by the convergent series $\sum \frac{1}{2^{k}}$, so $u$ is well defined and $u \in A$. Moreover, $u$ is easily seen to be a function in $P_{x_{0}}$.

Put $g=(1 / \lambda) f u$. To verify $g \in P_{x_{0}}$, pick $x \in X$. If $x \in F_{0}$, then we have

$$
|g(x)|=\frac{1}{|\lambda|}|f(x)|\left|u_{0}(x)\right| \sum_{k=1}^{\infty} \frac{\left|u_{k}(x)\right|}{2^{k}}<\frac{1}{|\lambda|}\|f\| \frac{|\lambda|}{\|f\|} \sum_{k=1}^{\infty} \frac{1}{2^{k}}=1 .
$$

If $x \in F_{n}$ for some $n \in\{1,2, \ldots\}$, then

$$
\begin{aligned}
|g(x)| & =\frac{1}{|\lambda|}|f(x)|\left|u_{0}(x)\right|\left(\frac{\left|u_{n}(x)\right|}{2^{n}}+\sum_{k \neq n} \frac{\left|u_{k}(x)\right|}{2^{k}}\right) \\
& \leq \frac{1}{|\lambda|}(|f(x)-\lambda|+|\lambda|)\left(\frac{\left|u_{n}(x)\right|}{2^{n}}+\sum_{k \neq n} \frac{1}{2^{k}}\right) \\
& <\frac{1}{|\lambda|}\left(\frac{|\lambda|}{2^{n}}+|\lambda|\right)\left(\frac{1}{2^{n}} \frac{1}{2^{n}+1}+1-\frac{1}{2^{n}}\right)=1 .
\end{aligned}
$$

If $x \in X \backslash \bigcup_{n=0}^{\infty} F_{n}$, then $f(x)=\lambda$ and so $g(x)=u(x) \in D_{1} \cup\{1\}$. Thus we obtain $g(X) \in D_{1} \cup\{1\}$. In particular, $g\left(x_{0}\right)=u\left(x_{0}\right)=1$. Hence $g \in P_{x_{0}}$, and the proof is completed.

Lemma 2.4. For $f, g \in A, f=g$ if and only if $(f u)(X)=(g u)(X)$ for all $u \in \mathcal{P}$.

Proof. The "only if" part is trivial. To show the "if" part, we assume $f \neq g$ and will find a $u \in \mathcal{P}$ such that $(f u)(X) \neq(g u)(X)$. Since $f \neq g$ on $X$ follows by $f \neq g$ on $\operatorname{Ch}(A)$, there is an $x_{0} \in \operatorname{Ch}(A)$ such that $f\left(x_{0}\right) \neq g\left(x_{0}\right)$. Without loss of generality, we can assume that $\left|f\left(x_{0}\right)\right| \leq\left|g\left(x_{0}\right)\right|$. 
If $f\left(x_{0}\right) \neq 0$, then Lemma 2.3 gives a $u \in P_{x_{0}} \subset \mathcal{P}$ such that $(1 / \lambda) f u \in P_{x_{0}}$, where $\lambda=f\left(x_{0}\right)$. Then $(f u)(X) \subset D_{|\lambda|} \cup\{\lambda\}$, while $(g u)\left(x_{0}\right)=g\left(x_{0}\right)$ and $g\left(x_{0}\right)$ cannot lie in $D_{|\lambda|} \cup\{\lambda\}$. Hence $(f u)(X) \neq(g u)(X)$.

On the other hand, if $f\left(x_{0}\right)=0$, then $g\left(x_{0}\right) \neq 0$. Put $r=\left|g\left(x_{0}\right)\right|$ and $F=\{x \in$ $X:|f(x)| \geq r\}$. Since $F$ is a closed subset of $X$ with $x_{0} \notin F$, there is a $u \in P_{x_{0}}$ such that $|u(x)|<r /(\|f\|+1)$ for every $x \in F$. It follows that

$$
|(f u)(x)|=|f(x)||u(x)| \begin{cases}\leq\|f\| \frac{r}{\|f\|+1}<r & \text { if } x \in F, \\ <r\|u\|=r & \text { if } x \in X \backslash F .\end{cases}
$$

Hence, for each $x \in X,|(f u)(x)|<r=\left|g\left(x_{0}\right)\right|=\left|(g u)\left(x_{0}\right)\right|$. This implies that $(f u)(X) \neq(g u)(X)$. Thus the lemma is proved.

\section{Proof of Theorem 1.1}

Put $\psi=T 1$, where 1 is the constant function which takes only the value 1 . We have $(T 1)^{2}(Y)=1^{2}(X)=\{1\}$, and so $\psi$ takes the value at most 1 or -1 .

Define the mapping $\widetilde{T}$ from $A$ into $B$ by

$$
(\widetilde{T} f)(y)=\psi(y)(T f)(y) \quad(y \in Y),
$$

for all $f \in A$. Then we easily check that $\widetilde{T}$ is a mapping from $A$ onto $B$ and satisfies

$$
\begin{gathered}
(T f)(y)=\psi(y)(\widetilde{T} f)(y) \quad(y \in Y, f \in A), \\
((\widetilde{T} f)(\widetilde{T} g))(Y)=(f g)(X) \quad(f, g \in A) .
\end{gathered}
$$

Moreover, we have $\widetilde{T} 1=1$ and so

$$
\widetilde{T} f(Y)=f(X) \quad(f \in A) .
$$

We show that there is a homeomorphism $\varphi$ from $M_{B}$ onto $M_{A}$ such that

$$
\widehat{\widetilde{T} f}(y)=\widehat{f}(\varphi(y)) \quad\left(y \in M_{B}\right) .
$$

It will follow that Theorem 1.1 holds.

Lemma 3.1. $\widetilde{T}$ is one-to-one.

Proof. Suppose $\widetilde{T} f=\widetilde{T} g$ for $f, g \in A$. Then, for each $u \in \mathcal{P}$, we apply (3.2) to see that

$$
(f u)(X)=((\widetilde{T} f)(\widetilde{T} u))(Y)=((\widetilde{T} g)(\widetilde{T} u))(Y)=(g u)(X) .
$$

By Lemma 2.4, we obtain $f=g$. Hence $\widetilde{T}$ is one-to-one.

Since $\widetilde{T}$ is a one-to-one mapping from $A$ onto $B$, we can consider its inverse $\widetilde{T}^{-1}$ from $B$ onto $A$. Clearly, $\widetilde{T}^{-1}$ has properties similar to $\widetilde{T}$ :

$$
\begin{gathered}
\left(\left(\widetilde{T}^{-1} f\right)\left(\widetilde{T}^{-1} g\right)\right)(X)=(f g)(Y) \quad(f, g \in B), \\
\left(\widetilde{T}^{-1} f\right)(X)=f(Y) \quad(f \in B) .
\end{gathered}
$$

Let $\operatorname{Ch}(B)$ denote the Choquet boundary of $B$. For each $y \in \mathrm{Ch}(B)$, put

$$
Q_{y}=\left\{v \in B: v(Y) \subset D_{1} \cup\{1\}, v(y)=1\right\}
$$

and $\mathcal{Q}=\bigcup_{y \in \operatorname{Ch}(B)} Q_{y}$. For each $h \in \mathcal{Q}$, we write $L_{h}=\{y \in Y: h(y)=1\}$.

Lemma 3.2. Let $f, g \in \mathcal{P}$. If $L_{\widetilde{T} f} \subset L_{\widetilde{T} g}$, then $K_{f} \subset K_{g}$. 
Proof. By (3.3), $f \in \mathcal{P}$ implies $\widetilde{T} f \in \mathcal{Q}$. Hence $L_{\widetilde{T} f}$ and $L_{\widetilde{T} g}$ is well defined. Suppose $L_{\widetilde{T} f} \subset L_{\widetilde{T} g}$ and assume that $u \in \mathcal{P}$ satisfies $1 \in(f u)(X)$. By (3.2), we have $1 \in((\widetilde{T} f)(\widetilde{T} u))(Y)$. For $\widetilde{T} u \in \mathcal{Q}$ and $1 \in((\widetilde{T} f)(\widetilde{T} u))(Y)$, we have that $1 \in((\widetilde{T} g)(\widetilde{T} u))(Y)$ by Lemma 2.2, and so $1 \in(g u)(X)$ by (3.2). We apply Lemma 2.2 again to conclude that $K_{f} \subset K_{g}$.

Lemma 3.3. For each $y \in \mathrm{Ch}(B)$, there exists an $x \in \mathrm{Ch}(A)$ such that $\widetilde{T}^{-1}\left(Q_{y}\right) \subset$ $P_{x}$.

Proof. Fix $y \in \mathrm{Ch}(B)$ and put $K=\bigcap\left\{K_{f}: f \in \widetilde{T}^{-1}\left(Q_{y}\right)\right\}$. We first observe that $K$ is nonempty. To show $K \neq \emptyset$, it is enough to see that the family $\left\{K_{f}\right.$ : $\left.f \in \widetilde{T}^{-1}\left(Q_{y}\right)\right\}$ has the finite intersection property. Pick $f_{1}, \ldots, f_{n} \in \widetilde{T}^{-1}\left(Q_{y}\right)$. Then $f_{i} \in \mathcal{P}$, for $\widetilde{T}\left(f_{i}\right)(Y)=f_{i}(X)$. Since $\widetilde{T}$ is onto, we find a $g \in A$ such that $\widetilde{T} g=\widetilde{T} f_{1} \cdots \widetilde{T} f_{n}$. Then $g$ is also an element of $\mathcal{P}$. Noting that $\widetilde{T} f_{1}, \ldots, \widetilde{T} f_{n} \in Q_{y}$, we easily see that $\widetilde{T} g \in Q_{y}$. Moreover we have $L_{\widetilde{T} g} \subset L_{\widetilde{T} f_{i}}$ for $1 \leq i \leq n$. For, if $(\widetilde{T} g)(z)=1$, then $\left(\widetilde{T} f_{1}\right)(z) \cdots\left(\widetilde{T} f_{n}\right)(z)=1$, and so $\widetilde{T} f_{1}(z)=\cdots=\widetilde{T} f_{n}(z)=1$, because each $\widetilde{T} f_{i}(Y) \subset D_{1} \cup\{1\}$. Thus Lemma 3.2 shows that $K_{g} \subset K_{f_{i}}$ for $1 \leq i \leq n$, namely $K_{g} \subset K_{f_{1}} \cap \cdots \cap K_{f_{n}}$. Here, since $\widetilde{T} g \in Q_{y}$, it follows from (3.3) that $1 \in(\widetilde{T} g)(Y)=g(X)$. Hence $K_{g}$ is nonempty and so is $K_{f_{1}} \cap \cdots \cap K_{f_{n}}$. Thus $\left\{K_{f} ; f \in \widetilde{T}^{-1}\left(Q_{y}\right)\right\}$ has the finite intersection property, and we conclude that $K$ is nonempty.

We next consider the restriction $\left.A\right|_{K}$ of $A$ to $K$. Since each set $K_{f}$ is a peak set, $K$ is a peak set in the weak sense. It follows from [1, Corollary 2.4.3] that $\left.A\right|_{K}$ is a uniform algebra on $K$. Thus the Choquet boundary of $\left.A\right|_{K}$ is not empty (see 1 , p. 93]). Take a point $x \in \operatorname{Ch}\left(\left.A\right|_{K}\right)$. Then $x$ is a peak point in the weak sense for $\left.A\right|_{K}$ and so for $A$ by [1, Corollary 2.4.4]. Thus $x \in K \cap \mathrm{Ch}(A)$.

Now choose $f \in \widetilde{T}^{-1}\left(Q_{y}\right)$ arbitrarily. Since $\widetilde{T} f \in Q_{y}$, it follows from (3.3) that $f(X)=(\widetilde{T} f)(Y) \subset D_{1} \cup\{1\}$. Also, we have $f(x)=1$, because $x \in K \subset K_{f}$. Hence $f \in P_{x}$. Thus we get $\widetilde{T}^{-1}\left(Q_{y}\right) \subset P_{x}$.

Lemma 3.4. For each $y \in \mathrm{Ch}(B)$, there corresponds a unique $x \in \operatorname{Ch}(A)$ such that $\widetilde{T}\left(P_{x}\right)=Q_{y}$.

Proof. Fix $y \in \mathrm{Ch}(B)$. By Lemma 3.3, there is an $x \in \mathrm{Ch}(A)$ such that $\widetilde{T}^{-1}\left(Q_{y}\right) \subset$ $P_{x}$. Since $\widetilde{T}^{-1}$ has the same properties as $\widetilde{T}$, we can apply Lemma 3.3 to $\widetilde{T}^{-1}$ and find a $y^{\prime} \in \operatorname{Ch}(B)$ such that $\widetilde{T}\left(P_{x}\right) \subset Q_{y^{\prime}}$. Hence

$$
Q_{y}=\widetilde{T}\left(\widetilde{T}^{-1}\left(Q_{y}\right)\right) \subset \widetilde{T}\left(P_{x}\right) \subset Q_{y^{\prime}} .
$$

Applying Lemma 2.1 we see that $y=y^{\prime}$, and $\widetilde{T}\left(P_{x}\right)=Q_{y}$.

To show the uniqueness of $x$, suppose that $\widetilde{T}\left(P_{x^{\prime}}\right)=Q_{y}$ for an $x^{\prime} \in \operatorname{Ch}(A)$. Then $\widetilde{T}\left(P_{x}\right)=Q_{y}=\widetilde{T}\left(P_{x^{\prime}}\right)$ and so $P_{x}=P_{x^{\prime}}$ because $\widetilde{T}$ is one-to-one. By Lemma 2.1, we get $x=x^{\prime}$.

According to Lemma 3.4, we associate to each $y \in \mathrm{Ch}(B)$ a point $x \in \mathrm{Ch}(A)$ with $\widetilde{T}\left(P_{x}\right)=Q_{y}$. This association gives the mapping $\phi$ from $\mathrm{Ch}(B)$ into $\operatorname{Ch}(A)$ such that

$$
\widetilde{T}\left(P_{\phi(y)}\right)=Q_{y} \quad(y \in \operatorname{Ch}(B)) .
$$


Lemma 3.5. For any $f \in A$ and $y \in \operatorname{Ch}(B),(\widetilde{T} f)(y)=f(\phi(y))$. In particular, $\widetilde{T}$ is an isometrical isomorphism from $A$ onto $B$.

Proof. Take $f \in A$ and $y_{0} \in \operatorname{Ch}(B)$ arbitrarily. Put $\alpha=f\left(\phi\left(y_{0}\right)\right)$ and $\beta=$ $(\widetilde{T} f)\left(y_{0}\right)$. We will show $\alpha=\beta$.

We first assume that $\alpha \neq 0$ and $\beta \neq 0$. Since $\alpha \neq 0$, Lemma 2.3 gives a $u \in P_{\phi\left(y_{0}\right)}$ such that $(1 / \alpha) f u \in P_{\phi\left(y_{0}\right)}$. By (3.4) $), \widetilde{T} u \in Q_{y_{0}}$ and $(\widetilde{T} u)\left(y_{0}\right)=1$. Also, we have $(f u)(X) \subset D_{|\alpha|} \cup\{\alpha\}$. Applying (3.2) we see that

$$
\begin{aligned}
\beta & =(\widetilde{T} f)\left(y_{0}\right) \\
& =(\widetilde{T} f)\left(y_{0}\right)(\widetilde{T} u)\left(y_{0}\right) \in((\widetilde{T} f)(\widetilde{T} u))(Y)=(f u)(X) \subset D_{|\alpha|} \cup\{\alpha\} .
\end{aligned}
$$

On the other hand, since $\beta \neq 0$, Lemma 2.3 gives a $v \in Q_{y_{0}}$ such that $(1 / \beta)(\widetilde{T} f) v \in$ $Q_{y_{0}}$. Since $\widetilde{T}\left(P_{\phi\left(y_{0}\right)}\right)=Q_{y_{0}}$, there is a $u \in P_{\phi\left(y_{0}\right)}$ such that $\widetilde{T} u=v$. Then $u\left(\phi\left(y_{0}\right)\right)=1$. Also, we have $((\widetilde{T} f) v)(Y) \subset D_{|\beta|} \cup\{\beta\}$. Hence we use (3.2) to see that

$$
\begin{aligned}
\alpha & =f\left(\phi\left(y_{0}\right)\right) \\
& =f\left(\phi\left(y_{0}\right)\right) u\left(\phi\left(y_{0}\right)\right) \in(f u)(X)=((\widetilde{T} f)(\widetilde{T} u))(Y) \\
& =((\widetilde{T} f) v)(Y) \subset D_{|\beta|} \cup\{\beta\} .
\end{aligned}
$$

Combining (3.5), we see that

$$
\beta \in D_{|\alpha|} \cup\{\alpha\} \quad \text { and } \quad \alpha \in D_{|\beta|} \cup\{\beta\},
$$

which forces that $\alpha=\beta$.

We next consider the case $\alpha=0$. Let $\varepsilon>0$ given and put $F=\{x \in X:|f(x)| \geq$ $\varepsilon\}$. Then $F$ is a closed set in $X$ and $\phi\left(y_{0}\right) \notin F$ because $f\left(\phi\left(y_{0}\right)\right)=0$. Hence there is a $u \in P_{\phi\left(y_{0}\right)}$ such that $|u(x)|<\varepsilon /(\|f\|+1)$ for every $x \in F$. By (3.4), $\widetilde{T} u \in Q_{y_{0}}$, and so $\widetilde{T} u\left(y_{0}\right)=1$. Also, we have $(f u)(X) \subset D_{\varepsilon}$, because

$$
|(f u)(x)|=|f(x)||u(x)| \begin{cases}\leq\|f\| \frac{\varepsilon}{\|f\|+1}<\varepsilon & \text { if } x \in F, \\ <\varepsilon\|u\|=\varepsilon & \text { if } X \backslash F .\end{cases}
$$

Hence

$$
\beta=(\widetilde{T} f)\left(y_{0}\right)=(\widetilde{T} f)\left(y_{0}\right)(\widetilde{T} u)\left(y_{0}\right) \in((\widetilde{T} f)(\widetilde{T} u))(Y)=(f u)(X) \subset D_{\varepsilon},
$$

that is, $|\beta|<\varepsilon$. Since $\varepsilon>0$ is arbitrary, we conclude that $\beta=0=\alpha$.

We finally consider the case $\beta=0$. We repeat the similar argument as above. Let $\varepsilon>0$ be given and put $F=\{y \in Y:|\widetilde{T} f(y)| \geq \varepsilon\}$. Then we can find a $v \in Q_{y_{0}}$ such that $|v(y)|<\varepsilon /(\|\widetilde{T} f\|+1)$ for $y \in F$. According to (3.4), take $u \in P_{\phi\left(y_{0}\right)}$ so that $\widetilde{T} u=v$. Then we have $u\left(\phi\left(y_{0}\right)\right)=1$ and see in the same way that $((\widetilde{T} f) v)(Y) \subset D_{\varepsilon}$. Hence we see that $\alpha \in D_{\varepsilon}$, which shows that $\alpha=0=\beta$.

In any case we see that $(\widetilde{T} f)(y)=f(\phi(y))$ holds. Since $g_{1}=g_{2}$ on $\mathrm{Ch}(B)$ implies $g_{1}=g_{2}$ on $X$ for any $g_{1}, g_{2} \in B$, we see that $\widetilde{T}$ is an isometrical isomorphism since $\mathrm{Ch}(B)$ is a boundary for $B$. 
Consider the adjoint operator $\widetilde{T}^{*}$ of $\widetilde{T}$. It is well known that $\widetilde{T}^{*}$ is a continuous mapping from $B^{*}$ into $A^{*}$, where $B^{*}$ and $A^{*}$ have the weak-star topology. Now let $\varphi$ be the restriction of $\widetilde{T}^{*}$ to $M_{B}$. Then we can see that $\widetilde{T}^{*}\left(M_{B}\right) \subset\left(M_{A}\right)$ and

$$
(\widehat{\widetilde{T}} f)(y)=y(\widetilde{T} f)=\left(\widetilde{T}^{*} y\right)(f)=\widehat{f}\left(T^{*} y\right)=\widehat{f}(\varphi(y))
$$

for all $y \in M_{B}$. Next, we repeat the above argument for $\widetilde{T}^{-1}$ and note that $\left(\widetilde{T}^{-1}\right)^{*}=\left(\widetilde{T}^{*}\right)^{-1}$. Then we know that $\varphi$ is a homeomorphism from $M_{B}$ onto $M_{A}$. It is easy to see that $\phi=\varphi$ on $\operatorname{Ch}(A)$. We see that

$$
\widehat{T f}(y)=\widehat{\psi \widetilde{T} f}(y)=\widehat{\psi} \widehat{f}(\varphi(y))
$$

holds for every $f \in A$ and $y \in M_{B}$.

Theorem 3.6. Let $X$ and $Y$ be compact Hausdorff spaces. If $T: C(X) \rightarrow C(Y)$ is a surjective mapping with the property that

$$
(\overline{T(f)} T(g))(Y)=\bar{f} g(X) \quad(f, g \in C(X)),
$$

then there exists a homeomorphism $\varphi: Y \rightarrow X$ and a function $\tau \in C(Y)$ of modulus 1 such that

$$
T(f)(y)=\tau(y) f(\varphi(y)) \quad(y \in Y, f \in C(X)) .
$$

Proof. Put $\tilde{T}(f)=\overline{T(1)} T(f)$ for every $f \in C(X)$. Since $|T(1)|=1$ holds for $\overline{T(1)} T(1)(Y)=1(X)=\{1\}$, it is easy to see that $\tilde{T}$ satisfies the condition in the theorem and $\tilde{T}(1)=1$. We also see by a simple calculation that $(\tilde{T}(f))(Y)=f(X)$ holds for every $f \in C(Y)$ and $\tilde{T}$ is a surjection from $C_{\mathbb{R}}(X)$ onto $C_{\mathbb{R}}(Y)$, where $C_{\mathbb{R}}(X)$ (resp. $C_{\mathbb{R}}(Y)$ ) is the algebra of all real-valued continuous functions on $X$ (resp. $Y$ ).

We show that $\tilde{T}$ is a surjective isometric isomorphism. First we prove that $(\bar{T}(f) \tilde{T}(g))(Y)=(\tilde{T}(\bar{f}) \tilde{T}(g))(Y)$ holds for every $f \in C(X)$ and $g \in C_{\mathbb{R}}(X)$. We see that $f g(X)=\tilde{T}(f) \tilde{T}(g)(Y)$ holds since $\bar{f} g(X)=\overline{\tilde{T}}(f) \tilde{T}(g)(Y)$ and $g \in C_{\mathbb{R}}(X)$. On the other hand

$$
f g(X)=\overline{(\bar{f})} g(X)=\overline{\tilde{T}(\bar{f})} \tilde{T}(g)(Y)
$$

holds, so we have $\bar{f} g(X)=(\tilde{T}(\bar{f}) \tilde{T}(g))(Y)$ for every $f \in C(X)$ and $g \in C_{\mathbb{R}}(X)$. Since $\tilde{T}$ is surjective on $C_{\mathbb{R}}(X)$ we have $(\tilde{\tilde{T}(f)} h)(Y)=(\tilde{T}(\bar{f}) h)(Y)$ for every $f \in$ $C(X)$ and $h \in C_{\mathbb{R}}(X)$. Fixing $f$ and varying $h$ we see that $\overline{\tilde{T}(f)}=\tilde{T}(\bar{f})$ holds. Thus we have

$$
(\tilde{T}(f) \tilde{T}(g))(Y)=\overline{(\tilde{T}(\bar{f})} \tilde{T}(g))(Y)=\overline{(\bar{f})} g(X)=f g(X)
$$

for every $f, g \in C(X)$. Then we see by Theorem 1.1 that $\tilde{T}$ is a surjective isometric isomorphism since $\tilde{T}(1)=1$. Thus there is a homeomorphism $\varphi: Y \rightarrow X$ such that $\tilde{T}(f)=f \circ \varphi$ for every $f \in C(X)$. Hence we have the conclusion since $T(f)=T(1) \tilde{T}(f)=T(1) f \circ \varphi$ for $|T(1)|=1$.

\section{REFERENCES}

[1] A. Browder, "Introduction to Function Algebras", W.A. Benjamin, 1969. MR0246125 (39:7431)

[2] S. Kowalski and Z. Słodkowski, A characterization of multiplicative linear functionals in Banch algebras, Studia Math., 67 (1980), 215-223 MR0592387|(82d:46070) 
[3] L. Molnár, Some characterizations of the automorphisms of $B(H)$ and $C(X)$, Proc. Amer. Math. Soc., 130(2002), 111-120 MR1855627 (2002m:47047)

[4] N. V. Rao and A. K. Roy, Multiplicatively spectrum-preserving maps of function algebras, Proc. Amer. Math. Soc., 133(2005), 1135-1142 MR2117215(2005k:46124)

Department of Mathematics, Faculty of Science, Niigata University, Niigata 9502181, JAPAN

E-mail address: hatori@math.sc.niigata-u.ac.jp

Department of Basic Technology, Applied Mathematics and Physics, Yamagarta UniVERSITY, YONEZAWA 992-8510, JAPAN

E-mail address: miura@yz.yamagata-u.ac.jp

Department of Mathematical Sciences, Faculty of Science, Shinshu University, MatSUMOTO 390-8621, JAPAN

E-mail address: takagi@math.shinshu-u.ac.jp 